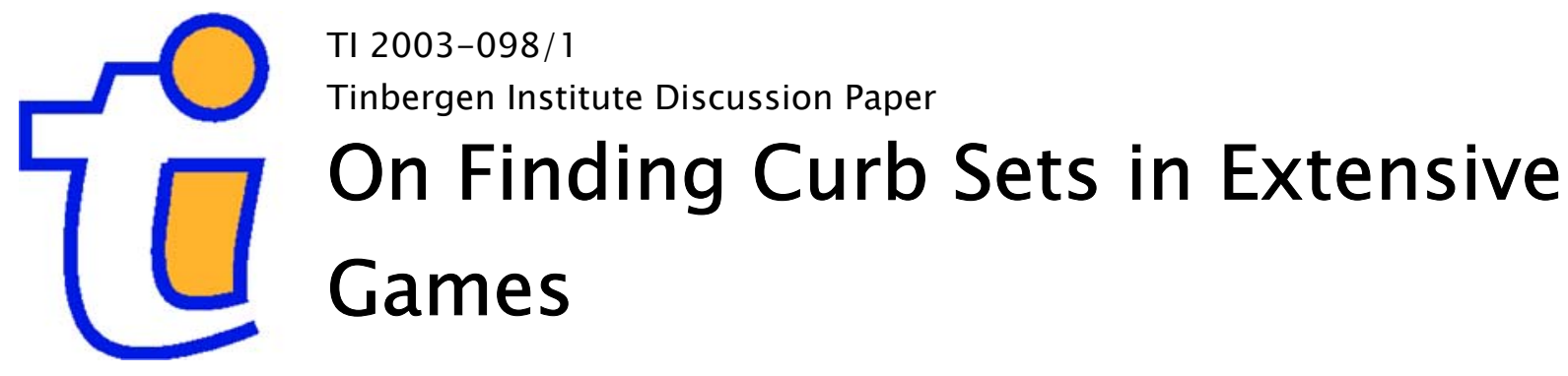

Vitaly Pruzhansky

Faculty of Economics, Vrije Universiteit Amsterdam, and Tinbergen Institute. 


\section{Tinbergen Institute}

The Tinbergen Institute is the institute for economic research of the Erasmus Universiteit Rotterdam, Universiteit van Amsterdam, and Vrije Universiteit Amsterdam.

Tinbergen Institute Amsterdam

Roetersstraat 31

1018 WB Amsterdam

The Netherlands

Tel.: $\quad+31(0) 205513500$

Fax: $\quad+31(0) 205513555$

Tinbergen Institute Rotterdam

Burg. Oudlaan 50

3062 PA Rotterdam

The Netherlands

Tel.: $\quad+31(0) 104088900$

Fax: $\quad+31(0) 104089031$

Please send questions and/or remarks of nonscientific nature to driessen@tinbergen.nl.

Most TI discussion papers can be downloaded at http://www.tinbergen.nl. 


\title{
On Finding Curb Sets in Extensive Games*
}

\author{
Vitaly Pruzhansky \\ FEWEB, Vrije Universiteit Amsterdam, De Boelelaan \\ 1105, $1081 \mathrm{HV}$ Amsterdam, the Netherlands
}

\begin{abstract}
We characterize strategy sets that are closed under rational behavior (curb) in extensive games of perfect information and finite horizon. It is shown that any such game possesses only one minimal curb set, which necessarily includes all its subgame perfect Nash equilibria. Applications of this result are twofold. First, it lessens computational burden while computing minimal curb sets. Second, it implies that the profile of subgame perfect equilibrium strategies is always stochastically stable in a certain class of games.
\end{abstract}

April 10, 2003

Keywords: rationalizability, stochastic stability.

\section{Introduction}

The concept of strategy sets that are closed under rational behavior (curb) was developed by Basu and Weibull [1] as a set-valued generalization of strict Nash equilibria. However, the practical application of these sets as solution concept faced a few significant difficulties. First, even relatively simple games tended to possess many such sets. Secondly, the latter could include non-equilibrium strategies. With the purpose of further narrowing down these sets, a concept of minimal curb (i.e. curb set that does not include any other curb as a proper subset of itself) was developed. Unfortunately, this did not solve the problem completely as non-equilibrium strategies could appear even in the minimal curb sets (for an intriguing example see the original paper [1]). Moreover, the

*I am grateful to J. Kamphorst, G. van der Laan and X. Tieman, who commented on the earlier versions of the paper. I also thank an anonymous referee and an associate editor for their helpful remarks. The usual disclaimer applies. 
computation of (minimal) curb sets remained quite a complex matter in games where the space of pure strategies was big or even infinite.

Originally Basu and Weibull developed this concept and provided examples only for games in normal from. In this paper we characterize curb sets in extensive form games of perfect information and finite horizon. Our main result is that for any game of an arbitrary complexity in this class minimal curb sets are easily computable, and necessarily contain all subgame perfect Nash equilibria. Apart from pure computational benefits associated with this finding, the result sheds some light on the process of equilibrium selection and stochastic stability of subgame perfect equilibria.

The remainder of the paper is organized as follows. Section 2 provides formal definitions and also states the results. Section 3 presents some applications.

\section{Computation of curb sets}

Our terminology on extensive games and subgame perfect equilibria is standard and follows Osborne and Rubinstein [5]. Throughout, the term extensive game applies to a game of perfect information and finite horizon, which is given by $\Gamma=\left\langle N, H, P,\left(\succeq_{i}\right)\right\rangle$, where

- $N=\{1, \ldots, n\}$ is a set of players.

- $H$ is a set of finite histories

$$
H:=\left\{h \mid \exists L \in \mathbb{N} \text { s.t. } h=\left\{a_{l}\right\}_{l=1}^{L}\right\},
$$

i.e. an element $h \in H$ is a sequence $\left\{a_{l}\right\}_{l=1}^{L}$ for some positive integer $L$. Each element of such a sequence is an action taken by a player. An empty sequence $\varnothing$ is also a member of $H$. A history $\left\{a_{l}\right\}_{l=1}^{L}$ is called terminal if $\nexists\left\{\widetilde{a}_{l}\right\}_{l=1}^{L+1} \in H$ with $\widetilde{a}_{l}=a_{l}$ for $l=1, \ldots, L$. The set of terminal histories is denoted by $Z$. To save space, we will write histories of length $L$ as $\{a\}^{L}$.

- $P$ is a player function, assigning a member of $N$ to each nonterminal history $h \in H \backslash Z$.

- $\succeq_{i}$ is a preference relation of player $i$ over the set of terminal histories $Z$.

For $h=\left\{a_{1}, \ldots, a_{L}\right\} \in H \backslash Z$ we denote the history $\left\{a_{1}, \ldots, a_{L}, a\right\}$ by $(h, a)$. After each $h \in H \backslash Z$ player $P(h)$ chooses an action from the set

$$
A(h)=\{a:(h, a) \in H\} .
$$


A strategy $s_{i}(h)$ of player $i$ in such a game is a function assigning to each $h \in H \backslash Z$ an action from the set $A(h)$, if and only if $P(h)=i$. The set of possible strategies for player $i$ will be denoted by $S_{i}$, and the strategy space of the game by the product

$$
S=\prod_{i=1}^{n} S_{i}
$$

For each profile of strategies $\mathbf{s}=\left(s_{1}, \ldots, s_{n}\right) \in S$ define the outcome $O(\mathbf{s})$ to be the terminal history that results after each $i$ plays $s_{i}$.

The definition of a subgame following the history $h, \Gamma(h)=\left\langle N,\left.H\right|_{h},\left.P\right|_{h},\left(\left.\succeq_{i}\right|_{h}\right)\right\rangle$, is similar to that of $\Gamma$, with the only difference that the empty (initial) history $\varnothing$ is replaced by $h$. Correspondingly, $\left.H\right|_{h}$ is a set of sequences $h^{\prime}$, such that $\left(h, h^{\prime}\right) \in H ;\left.P\right|_{h}$ is defined for each $\left.h^{\prime} \in H\right|_{h}$. Preference relations $\left(\left.\succeq_{i}\right|_{h}\right)$, strategies $\left.s_{i}\right|_{h}$ and outcomes $O_{h}$ are accordingly redefined. A subgame perfect equilibrium of $\Gamma=\left\langle N, H, P,\left(\succeq_{i}\right)\right\rangle$ is a strategy profile $\widehat{\mathbf{s}}=\left(\widehat{s}_{1}, \ldots, \widehat{s}_{n}\right)$, such that

$$
\left.O_{h}\left(\left.\widehat{s}_{i}\right|_{h},\left.\widehat{s}_{-i}\right|_{h}\right) \succeq_{i}\right|_{h} O_{h}\left(\left.s_{i}\right|_{h},\left.\widehat{s}_{-i}\right|_{h}\right)
$$

for any $i \in N, h \in H \backslash Z$ and any strategy $s_{i}$ of player $i$ in the subgame $\Gamma(h)$. Typically this equilibrium is found by the procedure termed backwards induction, which determines a subgame perfect equilibrium action $\left.\widehat{a}_{i}\right|_{h}$ for each player $i$ after every history $h \in H \backslash Z$ such that $P(h)=i$.

One genericity assumption that is commonly made in extensive games of perfect information is that no player is indifferent between any two terminal histories. This ensures that the subgame perfect equilibrium is unique. We do not employ this assumption here because it turns out that minimal curb sets have the most interesting configuration not when there is a unique subgame perfect equilibrium $\widehat{\mathbf{s}}$, but when it is strict (i.e. when for each player $i, \widehat{s}_{i}$ is the unique best reply to $\widehat{s}_{-i}$ ). Example in Section 3 clarifies this point.

The set of player $i$ 's best replies to the strategy profile $\mathbf{s}^{0}$ is given by all $s_{i}^{1} \in S_{i}$ enjoying the following property

$$
O\left(s_{i}^{1}, s_{-i}^{0}\right) \succeq_{i} O\left(s_{i}, s_{-i}^{0}\right)
$$

for all $s_{i} \in S_{i}$. If for some $s_{i}^{1} \in S_{i}$ this property holds for all $\mathbf{s}^{0} \in X \subset S$, then we will say that such $s_{i}^{1}$ is a best reply of $i$ to $X$. We denote this set by $\beta_{i}(X)$. Similarly,

$$
\boldsymbol{\beta}(X)=\prod_{i=1}^{n} \beta_{i}(X),
$$


is a set of all players' best replies to $X$. Such best reply operation can also be applied iteratively. For instance

$$
\beta(\beta(X))=\beta^{2}(X) .
$$

We will say that $\widehat{\boldsymbol{\beta}}^{k}(X)$ is a set of iterative best replies of order $k$ to $X$, and for $k \geq 2$ define

$$
\widehat{\boldsymbol{\beta}}^{k}(X)=\bigcup_{l=1}^{k} \beta^{l}(X) .
$$

By convention, $\beta^{1}(X) \equiv \beta(X)$.

The standard definition for $X$ to be a curb set is

$$
\boldsymbol{\beta}(X) \subset X .
$$

In words, the set of strategy profiles $X$ is said to be closed under rational behavior if and only if it contains its own best replies. However, for the purposes of this paper we will use an alternative definition. We will say that $\mathbf{s}^{1}$ is a best reply to $\mathbf{s}^{0}$, and write $\mathbf{s}^{1} \in \boldsymbol{\beta}\left(\mathbf{s}^{0}\right)$, if for each $i \in N$ we have

$$
O\left(s_{i}^{1}, s_{-i}^{0}\right) \succeq_{i} O\left(s_{i}, s_{-i}^{0}\right),
$$

for all $s_{i} \in S_{i}$. If $X$ is curb, then for any $\mathbf{s}^{0} \in X$ and $\mathbf{s}^{1} \in \boldsymbol{\beta}\left(\mathbf{s}^{0}\right)$ one must have $\mathbf{s}^{1} \in X$.

The curb set $X$ is said to be minimal if there is no other curb set $X^{\prime}$, such that $X^{\prime} \subset X$. With this background we can derive the first result.

Proposition 1 A subgame perfect equilibrium profile of strategies $\widehat{\mathbf{s}}$ is a member of all curb sets of $\Gamma=\left\langle N, H, P,\left(\succeq_{i}\right)\right\rangle$.

Proof. We prove the proposition by showing that given any initial profile of strategies $\mathbf{s}^{0}$, it is possible to construct a set of iterative best replies $\widehat{\boldsymbol{\beta}}^{k}\left(\mathbf{s}^{0}\right)$, that necessarily includes $\widehat{\mathbf{s}}$.

For a terminal history $h=\{a\}^{L} \in Z$ take the history of length $L-1$. Denote the set of all such 'shortened' histories by $H^{Z-1}$. For each $h \in H^{Z-1}$ consider $\mathbf{s}^{1}$ which is different from $\mathbf{s}^{0}$ only in the fact that $i=P(h)$ plays $\left.\widehat{a}_{i}\right|_{h}$, i.e. the subgame perfect equilibrium action induced by $\widehat{s}_{i}$, following the history $h$. Clearly, such $\mathbf{s}^{1} \in \boldsymbol{\beta}\left(\mathbf{s}^{0}\right)$, since no player can hurt himself by playing a subgame perfect equilibrium action immediately before the terminal history. In case $\mathbf{s}^{0}$ turns out to be equal to $\mathbf{s}^{1}$, redefine $\mathbf{s}^{1}:=\mathbf{s}^{0}$.

Now for each $h \in H^{Z-1}$ of length $L-1$ take the history of length $L-2$ and denote their set by $H^{Z-2}$. As an initial vector consider $\mathbf{s}^{1}$. Another vector $\mathbf{s}^{2}$, that is different from $\mathbf{s}^{1}$ only in the fact that all $i=P(h)$ play in $\mathbf{s}^{2}$ their backward induction action $\left.\widehat{a}_{i}\right|_{h}$ after each history $h \in H^{Z-2}$, 
is clearly a best response to $\mathbf{s}^{1}$, i.e. $s^{2} \in \boldsymbol{\beta}\left(\mathbf{s}^{1}\right)$. If $\mathbf{s}^{1}$ is equal to $\mathbf{s}^{2}$, redefine $\mathbf{s}^{2}:=\mathbf{s}^{1}$.

Continuing in this fashion, taking histories of shorter length at each step, in finite time (since the longest history is finite) we will end up in a state where subgame perfect equilibrium actions are played after every history. This is identical to saying that all players $i \in N$ are playing their subgame perfect equilibrium strategies $\widehat{s}_{i}$.

This logic is correct for any initial vector $\mathbf{s}^{0}$ and any vector of subgame perfect equilibrium strategies $\widehat{\mathbf{s}}$. Thus is it also correct for any member of any curb set. As long as at least one curb set always exists, as was proven by Basu and Weibull [1], the desired result follows.

The next result is on the number of minimal curb sets in $\Gamma=$ $\left\langle N, H, P,\left(\succeq_{i}\right)\right\rangle$.

Proposition 2 There exist only one unique minimal curb set $\widehat{X}$ in any extensive game of perfect information.

Proof. Let us construct a minimal curb set $\widehat{X}$ in $\Gamma$. By Proposition 1, $\widehat{X}$ must contain all subgame perfect equilibrium profiles of strategies $\widehat{\mathbf{s}}$. By the definition of curb set, however, it also should include all sets of iterative best replies to $\widehat{\mathbf{s}}$. Suppose we have found minimal $k \in \mathbb{N}$, such that $\widehat{\boldsymbol{\beta}}^{k}(\widehat{\mathbf{s}})$ is curb. By construction this curb set is minimal. Its uniqueness follows from the fact that any other curb set $X^{\prime}$ must also include the same set of best replies $\widehat{\boldsymbol{\beta}}^{k}(\widehat{\mathbf{s}})$, plus some other strategy vectors that belong to $\cup_{l=k}^{\infty} \boldsymbol{\beta}^{l}(\widehat{\mathbf{s}})$. However, as long as $\widehat{\boldsymbol{\beta}}^{k}(\widehat{\mathbf{s}})$ is curb, it will follow that $X^{\prime}$ contains a curb set as a subset of itself. Thus such $X^{\prime}$ cannot be minimal.

Throughout the proofs we maintain that the game is finite (has finite number of histories). It is clear that games of this type possess subgame perfect equilibria, thus the existence of $\widehat{\mathbf{s}}$ was taken for granted. However, the results are also valid for infinite games of finite horizon, provided their subgame perfect equilibria can be found by backwards induction.

\section{Applications}

One application of this result is the computational simplicity of finding minimal curb sets. As was stressed at the beginning, in normal form games (minimal) curb sets may include even non-equilibrium strategies. Therefore for arbitrary games with many pure strategies, finding curb sets may turn out to be a quite complex exercise. This is no longer the case once extensive games of perfect information are concerned. One 
just has to check if the profile of subgame perfect equilibrium strategies $\widehat{\mathbf{s}}$ is strict. If it is so, then $\{\widehat{\mathbf{s}}\}$ is a unique minimal curb set, because it already contains its own best replies, and any other curb set will contain $\{\widehat{\mathbf{s}}\}$ as a subset of itself. If, however, $\widehat{\mathbf{s}}$ is not strict, one needs to solve

$$
\min _{k \in \mathbb{N}} k \text {, such that } \widehat{\boldsymbol{\beta}}^{k}(\widehat{\mathbf{s}}) \text { is curb. }
$$

By Proposition $2, \widehat{\boldsymbol{\beta}}^{k}(\widehat{\mathbf{s}})$ will be a unique minimal curb set.

Below we show how to compute a minimal curb set in the two period version of the centipede game $\Gamma$, taken from Reny [7].

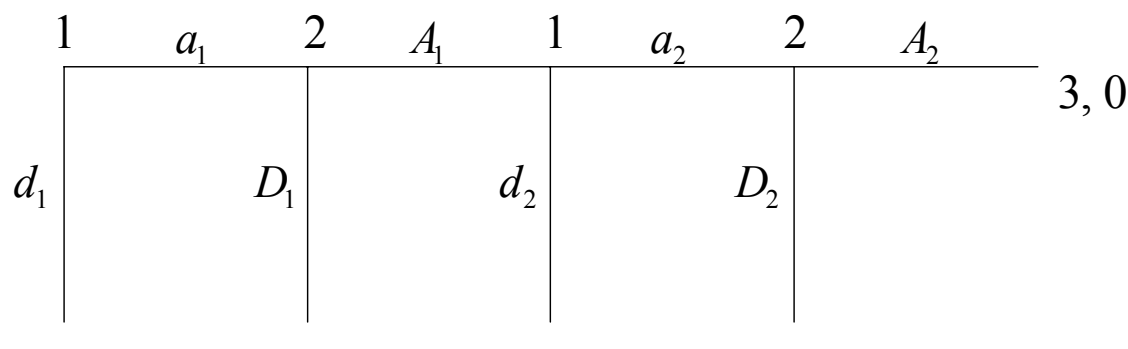
2,0
0,1
1,0
0,2

Fig.1: game $\Gamma$

The unique subgame perfect equilibrium of $\Gamma$ is given by the following profile $\widehat{\mathbf{s}}=\left(\left\{d_{1} d_{2}\right\},\left\{D_{1} D_{2}\right\}\right)$. This equilibrium is not strict, as none of player 2's information sets is reached. Thus any strategy yields him the same payoff, and is a best reply to player 1's equilibrium strategy $d_{1} d_{2}$. Furthermore, given $D_{1} D_{2}$, player 1 has two best replies: $d_{1} d_{2}$ and $d_{1} a_{2}$. Hence, $\boldsymbol{\beta}^{1}(\widehat{\mathbf{s}})=\left\{d_{1} d_{2}, d_{1} a_{2}\right\} \times\left\{D_{1} D_{2}, D_{1} A_{2}, A_{1} D_{2}, A_{1} A_{2}\right\}$. Obviously $\boldsymbol{\beta}^{1}(\widehat{\mathbf{s}})$ is not curb, because there are strategies of player 1 , that are best replies to some members of $\left\{D_{1} D_{2}, D_{1} A_{2}, A_{1} D_{2}, A_{1} A_{2}\right\}$, but are not in $\left\{d_{1} d_{2}, d_{1} a_{2}\right\}$. It is easy to check that at the next iteration we will have $\beta^{2}(\widehat{\mathbf{s}})=\left\{d_{1} d_{2}, d_{1} a_{2}, a_{1} a_{2}\right\} \times\left\{D_{1} D_{2}, D_{1} A_{2}, A_{1} D_{2}, A_{1} A_{2}\right\}$. The union of $\beta^{1}(\widehat{\mathbf{s}})$ and $\beta^{2}(\widehat{\mathbf{s}})$ gives us a complete strategy space, excluding the strategy $a_{1} d_{2}$ of player 1 , which is dominated by either $d_{1} d_{2}$ or $d_{1} a_{2}$. This set $\widehat{\beta}^{2}(\widehat{\mathbf{s}})$ is already curb, both maximal and minimal.

The way we have found the minimal curb set in the above game may appear similar to the procedure of finding rationalizable strategy profiles in the reduced strategic form of $\Gamma$. However, this similarity is deceptive. Although in this example indeed the minimal curb set and the set of rationalizable strategies are the same, in general this is not the case, and typically the former would be a subset of the latter. It is also worth stating that curb sets have even looser relation to rationalizability in 
extensive form, as defined in the sense of Pearce [6] and Battigalli [2]. There not every curb strategy can even be rationalizable. For instance, in $\Gamma$ the set of rationalizable strategies is just $\left\{d_{1} d_{2}, d_{1} a_{2}\right\} \times\left\{A_{1} D_{2}\right\}$, (see $[7]$ ).

Another application of Propositions 1 and 2 is that they sharpen the selection of equilibria in games played by boundedly rational individuals, who possess limited information about each other's actions and myopically choose best replies, occasionally making random mistakes. One variant of this behavioral procedure is termed adaptive play, and was introduced by Young in his seminal Econometrica paper [8]. To determine which strategies emerge or not wiped out in the long run (i.e. stochastically stable), one needs to focus on the convergence of adaptive play without mistakes. There is a general result in Young [9], (Theorem 7.2) that adaptive play without mistakes converges to minimal curb sets of a given game. It is also proven that only minimal curb sets can potentially be stochastically stable.

The fact that there is only one minimal curb set in a certain class of games is then of crucial importance and asserts two things. First, this minimal curb set is the only candidate for stochastic stability. Combining this with the existence theorem in [8], stating that a stochastically stable set always exists, makes the whole process of equilibrium selection a trivial exercise. Moreover, this holds true not only for adaptive play in the sense of Young, but for any other behavior or learning process, whose unperturbed dynamics converges to minimal curb sets. Secondly, the profile of subgame perfect equilibrium strategies $\widehat{\mathbf{s}}$ belongs to this curb set, and hence is always stochastically stable. This is in accordance with other equilibrium selection literature on extensive games of perfect information, where it was shown that for somewhat different learning processes $\widehat{\mathbf{s}}$ always belongs to the evolutionary stable set (Nöldeke, Samuelson [4], Hart [3] ). A somewhat disappointing result, though, is that despite the fact that subgame perfect equilibria are always stochastically stable, other strategies contained in the minimal curb set also are. The whole prediction of the game may therefore be quite loose. For instance, in the centipede game described above any strategy of player 1 except the dominated $a_{1} d_{2}$ and all player 2's strategies are in the minimal curb set, and, therefore, are stochastically stable.

\section{References}

[1] Basu, K., J. Weibull (1991) Strategy Subsets Closed Under Rational Behavior. Economic Letters 36: 141-146. 
[2] Battigalli, P. (1997) On Rationalizability in Extensive Games. Journal of Economic Theory 74: 40-61.

[3] Hart S. (2002) Evolutionary Dynamics and Backward Induction. Games and Economic Behavior 41: 227-264.

[4] Nöldeke, G., L. Samuelson (1993) An Evolutionary Analysis of Backward and Forward Induction. Games and Economic Behavior 5: 425454.

[5] Osborne, M.J., A. Rubinstein (1994) A Course in Game Theory, MIT Press.

[6] Pearce, D. (1984) Rationalizable Strategic Behavior and the Problem of Perfection. Econometrica 52: 1029-1050.

[7] Reny, Ph. (1992) Backwards Induction, Normal Form Perfection and Explicable Equilibria. Econometrica 60: 627-649.

[8] Young P. (1993) Evolution of Conventions. Econometrica 61: 57-84.

[9] Young P. (1998) Individual Strategy and Social Structure, Princeton Univ. Press. 